\title{
METALLICITY OF NGC 5128 GLOBULAR CLUSTERS
}

JABLONKA P.@1, BICA B.@2, PELAT D.@1, ALLOIN D.@1 @1 D.A.E.C, Observatoire de Paris, France @2 IF-UFRGS, Porto Alegre, Brazil

We have analyzed a selection of globular clusters based on the indications of high metallicity from previous photometric studies.

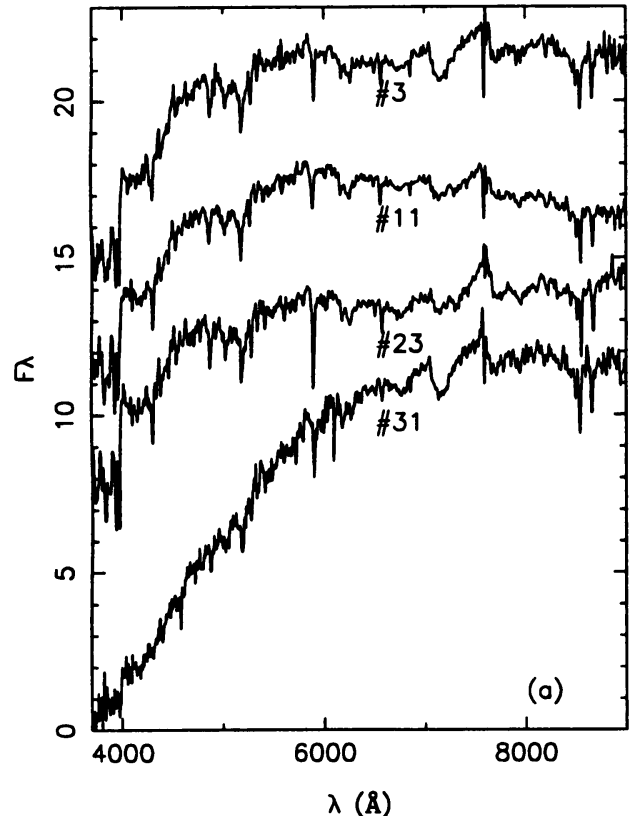

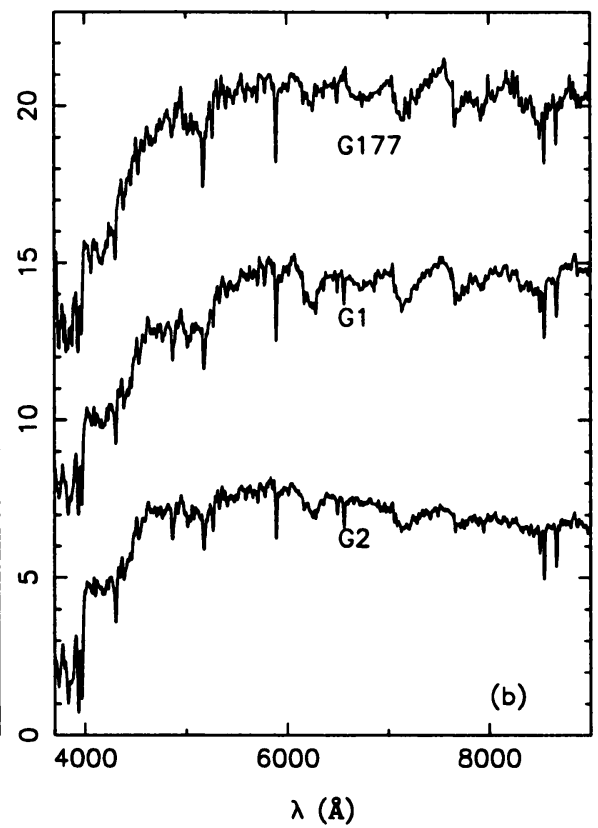

Figure 1. In (a), NGC 5128 star clusters observed in April 1993 at the ESO $3.6 \mathrm{~m}$ telescope. For the clarity of the figure only 4 of the 6 observed clusters have been plotted. In (b), the templates of comparison. G177 is located in the bulge of M31 and has a metallicity $\sim 3$ times solar. G1 is the representative template of solar metallicity clusters in our Galaxy. G2 is the template of Galactic globular clusters with metallicity $\sim 0.3$ time solar. All cluster spectra are normalized at $5870 \AA$ and shifted by some arbitrary constants for the purpose of the display. None of these globular clusters in NGC 5128 have spectra as strong-lined as G177 in M31. 